\title{
Analyser ses implications dans I'institution scientifique: une voie alternative
}

\section{Analisar as implicações na instituição científica: uma via alternativa}

\section{Analysing the involvements on the scientific institution: one alternative}

\section{Gilles Monceau}

Professor do Departamento de Ciências da Educação e coordenador do L'Axe socioclinique institutionnel de l'équipe de recherche ESSI, Universidade de Paris 8, Paris, França

\section{RESUME}

L'article interroge la possibilité, pour l'activité de recherche, de trouver une alternative en dehors de l'institution scientifique. La production des résultats de recherche est un travail institutionnel qui suppose le maniement de diverses techniques mais également d'agir sur l'institution. Le chercheur est impliqué dans l'institution, c'est-à-dire qu'il entretient avec elle des relations qui sont aussi bien idéologiques, organisationnelles que libidinales. En conséquence, c'est en analysant ses implications dans l'institution scientifique que le chercheur peut trouver une ligne de fuite afin de tenter d'échapper à la pensée instituée. René Lourau a proposé cette voie, l'article montre de quelle manière.

Mots clefs : Analyse institutionnelle, Implication, Institution.

\section{RESUMO}

O artigo interroga a possibilidade, para a atividade de pesquisa, de encontrar uma alternativa fora da instituição científica. A produção de resultados de pesquisa é um trabalho institucional que supõe o manejo de diversas técnicas, mas igualmente supõe um agir sobre a instituição. 0 pesquisador está implicado na instituição, ou seja, mantém com ela relações que são tanto ideológicas e organizacionais como libidinais. Conseqüentemente, é mediante a análise de suas implicações na instituição científica que o pesquisador pode encontrar uma linha de fuga, na tentativa de escapar ao pensamento instituído. René Lourau propôs esta via, e o artigo mostra de que maneira.

Palavras-chave: Análise institucional, I mplicação, Instituição.

\section{ABSTRACT}

The article asks the possibility for the research activity to find an alternative outside the scientific institution. The production of search results is an institutional work that involves the management of different techniques, but also implies acting on the institution. The researcher is involved in the institution, i.e. maintains relationships with it that are both ideological and 
organizational as libidinous. Therefore, it is through the analysis of his involvement in scientific institution that the researcher can find a line of escape, in an attempt to bolt from the instituted thought. René Lourau proposed this path, and the article shows in which way.

Keywords: Institutional analyse, Involvement, Institution.

Est ce qu'il existe des manières "alternatives" de faire de la recherche? Faut il développer, ailleurs que dans les institutions légitimées par l'Etat, des manières de produire des connaissances qui nous fassent échapper à l'institué scientifique? Finalement est ce qu'il existe une méthodologie de recherche hétérotopique (FOUCAULT, 2009), pouvant être développée en d'autres lieux que dans ceux consacrés par l'histoire et le pouvoir? René Lourau a répondu à cette question par une exigence: l'analyse de l'implication. Mais cette réponse est aussi issue d'un constat simple: on n'échappe pas à l'institution.

\section{Vivre les (des) institutions}

Pour quiconque veut mieux comprendre les institutions, rien ne sert de développer en dehors d'elles des alternatives plus conformes à ses désirs. Pour agir sur les institutions, il faut travailler à partir de ce qui nous relie à elles, nos implications.

Les institutions sont avec nous en tous lieux car nous les transportons dans notre subjectivité même. Sans l'institution, l'homme chute comme une marionnette sans fils ou bien, pour prendre une image différente, comme un corps sans squelette. L'institué, n'est pas seulement ce qui nous empêche, nous contraint ou nous oppresse, c'est aussi ce qui nous tient debout et nous relie aux autres. Ces autres qui se débattent, comme nous, dans leur attachement/répulsion vis-à-vis des institutions.

Le XIXème siècle et ses utopies (CABET, 1842) nous a bien montré qu'aller vivre ailleurs, en d'autres lieux, pour y fonder une autre société bâtie sur une autre manière de partager le travail et les ressources, ne permet pas d'échapper à la fatalité institutionnelle. On ne fuit pas les institutions. L'homme emporte avec lui ses "pathologies institutionnelles". Les indigènes des continents colonisés en ont fait l'expérience avec l'arrivée des européens, nouveaux venus, bâtisseurs utopistes ou conquérants.

Nous ne pouvons pas nous arracher à l'institution, nous l'emmenons avec nous, même lorsque nous la fuyons, mais nous avons aussi une forte tendance à l'imposer aux autres, " aux sauvages" " qui ne nous demandaient pourtant rien.

Les exemples de rebelles, de révolutionnaires, qui apportent leur énergie à la restauration de l'institution qu'ils ont cru, un temps, combattre, ne manquent pas non plus. Que les institutions changent de noms dans les périodes révolutionnaires ne change rien à l'affaire. 
L'énoncé simple selon lequel l'institutionnalisation transforme l'instituant (négation de l'institué) en son contraire reste tout simplement vrai.

Tenter de transformer l'institution, c'est donc nécessairement le faire de l'intérieur, débarrassé de l'illusion d'un ailleurs ou s'inventerait une vie sociale radicalement différente.

\section{Travailler l'institution}

L'institution n'est donc ni bonne ni mauvaise, elle est. Si nous n'existons qu'avec elle, elle n'existe qu'avec nous. Une institution disparaît si nous n'y croyons plus. C'est le cas d'anciennes religions ou de systèmes matrimoniaux dépassés par les conditions de vie modernes qui dispersent les familles. Car malgré leurs forces, et bien qu'elles imprègnent nos pratiques (MONCEAU, 2008a) en profondeur, les institutions se transforment et semblent parfois même mourir. Cependant, comme l'a montré René Lourau, elles renaissent et se prolongent sous d'autres formes (LOURAU, 1980).

L'analyse institutionnelle trouve son origine dans ce travail de l'institution, de l'intérieur. L'hôpital psychiatrique est ainsi devenu, à partir de la seconde guerre mondiale, le lieu du travail de la psychothérapie institutionnelle par lequel les hommes ont agi en travaillant d'abord sur eux mêmes.

Les psychiatres Daumezon, Bonnafé ${ }^{2}$, Tosquelles et Oury n'ont pas nié l'existence de l'institution psychiatrique mais ils y ont développé d'autres manières de faire. Jean Oury lui-même, qui pourtant quitte I'hôpital public au début des années 1960 pour créer une clinique (MICHAUD, 1977) qui fonctionnera autrement, sait bien qu'il emporte avec lui des rapports de pouvoir institués entre malades, infirmiers et médecins. C'est dans les actes quotidiens, dans des dispositifs sans cesse retravaillés, que ces praticiens travaillent l'institution en menant simultanément son analyse et sa réforme.

On ne se déprend pas de l'institution comme on le ferait d'un envoûtement, à moins d'être soi-même son propre désenvoûteur. C'est en construisant et en modifiant des dispositifs, dont des agencements du temps, de l'espace, des actes et des relations que l'on travaille l'institution. II en va de la recherche comme des autres pratiques sociales: nous ne pouvons changer l'institution qu'en changeant notre manière de la vivre, d'y être impliqué (MONCEAU, 2008b).

\section{Des voies alternatives qui ne nient pas leurs implications dans l'institution}

En 1964, dans I'un de ses premiers articles (LOURAU, 1964), publié dans une revue anarchiste, Lourau alors professeur de français, présente une étude sur différents mouvements poétiques ayant tenté de révolutionner le langage en s'attaquant à la grammaire. Ce texte 
porte déjà en lui une manière de concevoir les rapports entre individus et institutions qui restera constante dans toute l'œuvre de Lourau (LAMI HI; MONCEAU, 2002).

II écrit:

\begin{abstract}
Il faut en convenir: le poète qui, par méfiance à l'égard du langage voulait violenter la cohérence du langage en s'attaquant d'abord à la logique, ensuite à la grammaire, et qui parfois poussait sa révolte jusqu'à vouloir se passer du langage, finit par retomber dans les chaînes impures des mots et de la logique. Ce renversement dialectique, presque tous les mouvements poétiques du siècle l'ont connu ou le connaissent. (...) Au terme de son pénible itinéraire, le poète qui sort du tunnel découvre en guise de lumière l'inflation verbale et les systèmes idéologiques. Autant dire qu'il est revenu à son point de départ. (LOURAU, 1964, p. 128)
\end{abstract}

Dans ce passage, Lourau formule la fatalité qui pèse sur toute tentative révolutionnaire. Cela préfigure ce qui deviendra le moment de l'institutionnalisation dans sa dialectisation du concept d'institution (LOURAU, 1975). Dans certaines de ses formulations les plus lapidaires, il définira l'institutionnalisation comme étant ce qui transforme l'instituant en son contraire. Cependant, comme on le voit dans la suite de l'article et tout particulièrement dans sa conclusion, il ne s'agit pas d'opposer le "bon individ" (ici le poète) et le "mauvais institué" (ici la structure du langage).

Le langage est tout d'abord posé comme étant "par excellence le lieu où s'opère l'échange entre les hommes", "la structure de tous les rapports humains". Ainsi, si le langage est

l'institution humaine primordiale, la poésie est du même coup authentifiée en tant que langage et en tant que recherche permanente d'un métalangage, c'est-à-dire d'une transparence et d'une sur-signification des mots. (LOURAU, 1964, p. 129)

Le poète est au langage, pour le Lourau de 1964, ce que le socianalyste sera à l'institution pour le Lourau de 1969.

Ce rapport du poète à l'institution est cependant à analyser plus finement:

A ce titre, le poète est au service d'une institution, et d'une institution dont il n'est pas le maître. Pour que dans son domaine il parvienne à établir des rapports toujours plus complets, toujours plus clairs entre les hommes, il se soumet à la structure de l'institution, c'est-à-dire à la structure du langage (grammaire, syntaxe). (LOURAU, 1964, p.129)

Ainsi, pour pouvoir "intervenir" sur et dans l'institution du langage, le poète doit donc se soumettre à l'institué du langage, la grammaire. 
Cette thématique de la soumission à l'institution reviendra régulièrement dans les textes de Lourau mais avec de nombreuses variantes, toujours pour complexifier cette soumission qui n'est jamais totale. C'est déjà le cas dans ce texte de 1964:

\begin{abstract}
A vrai dire il est faux de parler de soumission, puisqu'il est lui-même le gardien et le dépositaire du langage. Mais il n'est pas seulement cela. L'erreur ou l'anachronisme de la poésie (aussi talentueuse ou géniale soit-elle) consiste à considérer que l'institution dont j'ai parlé est isolée parmi les autres institutions, ou très différente de ces autres institutions. C'est l'erreur de croire au langage, chose belle. Respecter la syntaxe est bien, est indispensable, dans la mesure où la syntaxe assure le fonctionnement normal et la valeur de l'institution langage. Mais qu'en est-il des autres institutions?, comment assurent-elles, ou n'assurent-elles pas la communication et un échange réel entre les hommes? (LOURAU, 1964, p. 129)
\end{abstract}

Le langage est donc une institution parmi les autres. Mais il est aussi la métaphore de toute institution. Lourau se demande ainsi ce qu'il en est de la "syntaxe", de la "grammaire" et des "structures" des autres institutions. Le renversement n'est pas anodin et on y voit à la fois les implications de l'auteur dans l'enseignement de la langue (il est alors professeur de français) et son passage de la littérature à la sociologie (il va préparer un doctorat en sociologie).

C'est enfin, l'idée selon laquelle l'existence d'une élite éclairée ne préfigure en rien le moindre changement pour "la masse":

Il est bon, il est magnifique que les poètes se parlent, se comprennent. Mais les autres? les autres hommes? se parlentils? et s'ils se parlent, s'entendent-ils, se répondent-ils ? Cette immense fraternité à laquelle aspirent les poètes par l'intercession du langage, peut-on croire avec Pierre Boujut $\left(\mathrm{TdF} \mathrm{n}^{\circ} 76\right)$ que la poésie finira par en faire cadeau aux autres institutions? ou bien faut-il croire que le salut est pour quelques-uns seulement, dans le ciel poétique, et que la masse ne cessera pas de s'enfoncer dans les malédictions dont notre époque semble à peine consciente? (LOURAU, 1975 p.129).

On retrouve ici une thématique, qui reviendra en 1969 dans les premières pages de L'instituant contre l'institué (LOURAU, 1969) puis qui fera l'objet du Lapsus des intellectuels (LOURAU, 1981), celle du rapport privilégié à l'institution qu'entretiennent des catégories d'individus qui en tirent avantage.

Ce développement de Lourau, mêlant langage et institution, évoque l'idée de Lacan selon laquelle "I'inconscient est structuré comme un 
langage", idée que le psychanalyste travaillera dans son séminaire "La logique du fantasme" en 1966-67. Le structuralisme imprègne alors très fortement les manières de penser des intellectuels et et la théorie émergente de l'analyse institutionnelle n'y échappe pas.

On y perçoit également, dès le départ, une charge politique (et poétique) dans la réflexion sur les rapports entre individu et institution, avant même que le concept d'implication ne soit formulé.

\title{
Le travail des contradictions
}

Lourau publie en 1964, un autre article dont le titre, "L'autogestion instituée" (LOURAU, 1971a) ${ }^{3}$, est tout à fait représentatif d'une approche dans laquelle l'analyse est portée par des contradictions actives. Dans ce texte, la contradiction s'exprime entre les institutions externe et interne (notions produites par Georges Lapassade). Le pédagogue autogestionnaire est membre de l'institution externe (l'institution scolaire), il est donc placé sous le contrôle des autres professeurs et des surveillants. Mais il est aussi membre de l'institution interne de la classe dans laquelle s'institue un fonctionnement autogéré. L'enseignant autogestionnaire se trouve dès lors considéré comme un déviant par les membres de l'institution externe (ses collègues) et reste "étranger" aux membres de l'institution interne (ses élèves) parce qu'ayant toujours la possibilité de reprendre le pouvoir et de mettre fin à l'expérience. Cette difficulté n'est cependant pas traitée comme rendant l'autogestion pédagogique impossible mais comme ouvrant à l'analyse de la contradiction institutionnelle plus large que perpétue l'institution scolaire : former des individus responsables en les privant de toutes responsabilités.

S'adressant visiblement aux parents de ses élèves, Lourau écrit:

\begin{abstract}
Je ne crains pas de choquer certains parents en disant que la pédagogie autoritaire, celle qu'ils ont connue, est peut-être capable de fabriquer des "grosses têtes" mais qu'elle échoue généralement à former des hommes. La véritable formation s'effectue alors en dehors de l'école, et parfois contre l'école. Pour ma part, j'ai choisi d'être avec mes élèves comme avec des êtres humains, et non comme avec des animaux que l'on gave et que l'on dresse. Notre époque exige des individus capables de s'adapter, de se diriger eux-mêmes, de ne pas rester dans la routine (c'est de plus en plus vrai dans le secteur industriel). Apprendre à s'exprimer, à comprendre les autres, à les écouter avant de leur répondre, discuter avant de juger, penser par soi-même, faire son auto-critique, prendre des initiatives: tels sont les buts de l'autogestion pédagogique. (LOURAU, 1971a, p.102)
\end{abstract}

$\mathrm{Ce}$ texte contraste avec d'autres écrits sur la pédagogie autogestionnaire, tendant à la présenter comme une démarche 
révolutionnaire plus ou moins destinée à détruire l'Ecole. Les implications enseignantes de Lourau parlent très fort ici et l'on peut même remarquer le fait qu'il ne craint pas de mobiliser le discours "moderniste" pour vanter les mérites de sa pédagogie. Un esprit mal intentionné pourrait lire dans cet extrait une tentative de présenter l'autogestion pédagogique comme le plus sûr moyen de former les cadres des entreprises industrielles performantes!

Dans un autre texte rédigé par Lourau publié dans le même ouvrage collectif (LOURAU, 1971b), le ton est différent. Ce sont les "implications politiques" (cette fois-ci au sens de conséquences) de la notion d'autogestion qui sont discutées. Dans ce texte qui passe en revue les différentes approches de l'autogestion, l'auteur fait d'abord remarquer que "le contexte de la formation, de l'animation et de la sociologie du développement" est marqué par une tendance à "dépolitiser les problèmes". II adopte donc la démarche inverse. II s'agit tout d'abord de situer l'articulation entre éducation et révolution:

La révolution n'est pas une affaire d'éducation, mais l'éducation, toute déterminée qu'elle soit par les conditions politiques du moment, agit en retour sur ses propres déterminations, ne serait-ce qu'en travaillant la contradiction entre projet révolutionnaire et pratique réformiste, c'est-àdire en mettant à jour l'écart entre le facteur subjectif et le facteur objectif dont est constitué tout processus révolutionnaire. (LOURAU, 1971b, p.19)

L'éducation dont il est question ici est bien sûr celle qui adopte la perspective autogestionnaire et qui initie ainsi une pratique analytique au sein de l'institution scolaire, dans la salle de classe: "Le contenu de cette analyse des contradictions que produit pour le moins l'autogestion, aussi timide soit-elle, est à préciser sur le plan théorique". (LOURAU, 1971b, p.20). Et c'est d'ailleurs dans un passage intitulé " Autogestion et analyse institutionnelle " qu'apparaît le concept d'implication institutionnelle, suite à un paragraphe où la jonction est faite entre autogestion, lutte des classes et analyse institutionnelle. La lutte des classes y est alors considérée comme le "sujet de l'histoire "et" le moteur caché des institutions et de l'organisation sociale". Lourau écrit en conséquence:

L'analyse institutionnelle, en effet, tente de dépasser et la psychosociologie des groupes et la sociologie des organisations en analysant les déterminations cachées des groupes comme des systèmes d'organisation. (LOURAU, 1971b, p.20) 
Il écarte ainsi à la fois la perspective de la sociologie des organisations de Michel Crozier et les techniques psychosociologiques suspectes de "psychologisation " donc de dépolitisation.

Au début des années 1970 et avec la fréquentation de Henri Lefebvre, le marxisme va un temps influencer la réflexion de Lourau, via la dimension matérialiste. Cependant, il s'agira pour lui, comme pour beaucoup d'autres à l'époque d'être plus marxistes que les marxistes en dénonçant régulièrement (c'est aussi le cas dans le texte en question) le fait que les organisations politiques se réclamant du marxisme se nourrissent de la révolution plus qu'elles n'y contribuent (SAVOYE, 1973). Ce faisant elles participent à obscurcir le " système institutionnel aux yeux des individus.

Lourau pose donc la nécessité de travailler les implications institutionnelles pour lutter contre cet état de fait:

\begin{abstract}
Mettre en question nos implications institutionnelles, c'est toujours proposer une conception des institutions en termes de régulation par la base, de critique permanente des normes instituées, de développement des forces instituantes. C'est démasquer le complot des institutions, qui consiste à imposer, par la contrainte matérielle (économique ou physique) et idéologique, une vision fausse des rapports de production. (LOURAU, 1971b, p. 22)
\end{abstract}

La rhétorique du complot, de la domination et de l'aliénation est très présente dans les années 1970 et l'analyse institutionnelle se présente donc comme une réponse.

Le mode éducatif promu par Lourau, l'autogestion, se propose ainsi de repolitiser les institutions éducatives non pas en faisant la promotion d'un dogme politique mais en luttant au quotidien contre toutes les formes de "complots" (de quelques origines idéologiques qu'ils viennent) visant à dissimuler les déterminations institutionnelles. Ce qui est énoncé ici est le fait que l'analyse des implications, comme voie privilégiée de l'analyse institutionnelle, doit rendre cette dernière irrécupérable par les organisations politiques. Par contre, une certaine complicité pourra exister avec la tradition anarchiste comme le montre l'assez bonne réception des travaux de Lourau dans ce milieu et comme le laissait déjà supposer la publication du "Poète devant les institutions" dans une revue anarchiste.

\title{
Analyse de l'implication et épistémologie
}

Chaque chapitre du livre Institution et implication, que j'ai coordonné avec Ahmed Lamihi, traitait d'un ouvrage de Lourau. J'ai travaillé pour ma part sur Actes manqués de la recherche. Dans cette contribution (MONCEAU, 2002), j'avais entrepris un premier bilan de mon appropriation de sa théorie de l'implication. En même temps, 
s'opérait un premier réaménagement de mon rapport aux travaux de Lourau. Dans ce texte, je soulignais qu'après avoir déjà utilisé les concepts psychanalytiques d'inconscient (LOURAU, 1978) et de lapsus (LOURAU, 1981) dans deux de ses livres précédents, il utilisait celui d'acte manqué. Je commentais ensuite le cheminement intellectuel allant de l'idée selon laquelle les intellectuels construisent leur statut en refusant l'analyse de leurs implications (LOURAU, 1981) à celle selon laquelle ces implications échappent en grande partie à la conscience du chercheur (LOURAU, 1994). D'une certaine façon, la proximité avec la démarche psychanalytique était alors assumée et il sera désormais moins question de "complot " que de construction de dispositifs susceptibles de permettre la prise de conscience. Dans le même texte, je faisais également remarquer que Lourau assumait une nouvelle posture en perdant certains de ses accents révolutionnaires pour en adopter de plus épistémologiques, ceci en dialoguant avec Auguste Comte, John Dewey, Lucien Levy-Bruhl, Sigmund Freud, mais aussi Jean Piaget.

Dans l'Analyse institutionnelle, Lourau s'appuie sur un texte de Freud "Psychologie collective et analyse du moi" dont il extrait la triade libidinal/organisationnel/idéologique qu'il utilisera pour caractériser la manière dont les groupes humains s'instituent puis pour décliner les trois types de déviance dans les institutions.

Dans ce texte, Freud donne la définition suivante du concept de libido:

Libido est un terme emprunté à la théorie de l'affectivité. Nous désignons ainsi l'énergie, considérée comme une grandeur quantitative -quoique pour l'instant non encore mesurable-, de ces pulsions qui ont affaire avec tout ce que nous résumons sous le nom d'amour. (FREUD, 1981, p. $150)^{4}$

Cette définition relativement extensive facilite sans doute l'appropriation du terme par le courant d'analyse institutionnelle en devenant un quasi synonyme de l'affectivité.

En menant la critique de Le Bon (1963) ${ }^{5}$ et de Mac Dougall (1920), Freud cherche à établir l'articulation entre la psychologie individuelle et la psychologie collective, en particulier en essayant de décrypter la manière dont certains chefs peuvent conduire les foules. L'observation de la modification de l'état de conscience et du comportement d'un individu lorsqu'il est en foule, mobilise les trois dimensions évoquées plus haut. Cette triade sera utilisée par la suite en analyse institutionnelle à la fois pour penser les différentes dimensions de l'institution et celles des différentes dimensions de l'implication des individus dans l'institution. 
Plus loin dans L'analyse institutionnelle, Lourau met en forme une définition de l'implication qui n'est pas sans faire écho à la sociologie des organisations ${ }^{6}$ :

\begin{abstract}
Ayant considéré la distance institutionnelle comme l'écart entre l'action et les bases rationnelles de cette action, on désignera par les termes d'implication institutionnelle l'ensemble des rapports existant, consciemment ou non, entre l'acteur et le système institutionnel. (LOURAU, 1975, p. $272-273)^{7}$
\end{abstract}

Dans L'analyse institutionnelle encore, Lourau définit ensuite I' "implication pratique" comme étant

un corollaire de la distance pratique. Si cette dernière mesure l'écart de l'acteur par rapport à la base rationnelle des techniques, l'implication pratique indique les rapports réels qu'il entretient avec ce que l'on a nommé plus haut la base matérielle des institutions. (LOURAU, 1975, p.273)

Cette implication pratique permettra de faire le lien entre les places successivement occupées par Lourau: praticien de l'enseignement primaire puis secondaire et enfin enseignant-chercheur à l'université. Quelle que soit cette place, il s'agit d'un "chercheur-praticien". Par contre les notions de "distance institutionnelle" et de "distance pratique" ne seront pas reprises par Lourau dans la suite de son parcours. Dans la mesure où l'implication allait désigner l'ensemble des rapports à l'institution, la "distance" allait nécessairement en devenir l'une des modalités.

Avec ces "implication institutionnelle" et "implication pratique", se dessinent les deux niveaux qui donneront naissance à la distinction entre implications primaires et implications secondaires. Mais pour I'heure, c'est une typologie complémentaire qui est avancée: les implications syntagmatique, paradigmatique et symbolique qu'il formule en empruntant à son directeur de thèse Henri Lefèbvre. Ce dernier, dans un ouvrage consacré à la sociolinguistique proposait un modèle tridimensionnel du signe linguistique (LEFEBVRE, 1966, p. 266). On peut voir dans cette circulation d'idées entre linguistique et sociologie une certaine résonance avec les intérêts de Lourau pour la littérature et les mouvements littéraires ${ }^{8}$. Cette proposition ne sera cependant pas reprise par la suite.

Notons que c'est dans son premier ouvrage publié L'instituant contre l'institué (LOURAU, 1969), qu'apparaît la définition de l'implication institutionnelle comme étant

la somme des appartenances (subjectives et objectives) et des références dans laquelle s'inscrit l'individu et ceci par opposition à la distance institutionnelle qui est posée 
comme la somme des non-appartenances (subjectives et objectives) et des non références dans laquelle il s'inscrit. (LOURAU, 1969, P. 14)

Ces définitions de l'implication et de la distance institutionnelles s'inspirent des concepts de "subréalismes" et de "surréalisme" de J. Gabel (1962). Le rapport "subréaliste" à l'institution se traduirait par une identification "aux normes, aux hiérarchies et aux personnes qui les incarnent" tandis que le rapport "surréaliste" serait caractérisé par un "défaut d'identification", un "réalisme morbide" et une "saisie trop structurée du réel". Mais il s'agit-là, pour l'auteur, de "types extrêmes" qui doivent être pris en compte dialectiquement et non dans une opposition binaire.

Dans le même passage, intitulé "Les particuliers comme usagers et mainteneurs des institutions" Lourau (1969, p.8-17) développe son approche du rapport entre les individus et les institutions. On y retrouve une idée déjà présente dans ses textes pédagogiques selon laquelle l'enseignant peut-être à la fois le serviteur de l'institué et le promoteur de l'instituant. Le même individu peut ainsi actualiser, dans sa vie privée ou professionnelle, les différents moments du concept d'institution.

Combien d'individus ventriloques ne parlent que parce que l'institution parle à travers eux, parce qu'ils l'ont littéralement "dans la peau" ${ }^{9}$ ! Mais aussi combien se refusent à adhérer aux institutions, alors que la vie quotidienne est tout entière composée d'un tissu institutionnel impliquant un certain degré de consentement, d'adhésion, d'engagement et de participation (sinon d'intégration) (LOURAU, 1969, p. 12) ${ }^{10}$

Nous sommes donc tous concernés par l'institution, même lorsque nous n'appartenons pas objectivement à sa forme instituée.

C'est ainsi que riche ou pauvre, salarié ou actionnaire, l'institution de la banque; et les organismes apparentés à la banque, me concernent: j'ai affaire à la banque, même si je n'ai pas de compte en banque, pas de livret de caisse d'épargne, pas de $C C P^{11}$, parce que la banque est la forme instituée de la régulation concernant la circulation et l'accumulation du capital (LOURAU, 1969, p. 10)

Dans une note bibliographique consacrée à cet ouvrage, parue dans la Revue française de sociologie ${ }^{12}$ en 1970, Alain Revon souligne à la fois son intérêt pour l'approche développée par Lourau et la (trop) grande diversité des terrains abordés. II conclut ainsi: “En réalité, il n'était pas possible de traiter à fond de sujets aussi variés que le surréalisme, le syndicalisme, la poésie et l'autogestion en moins de 
200 pages". Le reproche d'être un "touche à tout" qui n'approfondit pas le travail de ses objets est donc formulé, difficile de faire alors entendre que ce ne sont pas ces objets en eux-mêmes qui intéressent l'auteur mais ce qu'ils peuvent avoir de transversal et qui pourrait apporter à une théorie (générale ?) de l'institution donc de l'implication.

Si L'instituant contre l'institué a pour sous titre “Essais d'analyse institutionnelle", ce qui évoque les Essais de psychanalyse ${ }^{13}$ de Freud, l'influence de la psychanalyse n'y est guère manifeste dans l'usage des concepts. On y trouve par contre un chapitre, "La psychanalyse dans la division du travail" (LOURAU, 1969), qui prend la psychanalyse elle-même comme objet d'analyse en insistant particulièrement sur son indifférence aux institutions:

\begin{abstract}
A ces institutions, qui vont du salariat et du profit à la hiérarchie et à l'Etat, en passant par bien d'autres, l'analyste n'accorde qu'une attention "flottante". En fait, dans la mesure où il intériorise lui-même les contraintes de l'ordre institué, en les assimilant au principe de réalité, il investit dans son analyse du contre-transfert un énorme point aveugle (...). (LOURAU, 1969, p.138)
\end{abstract}

Et Lourau développe son point de vue en s'appuyant sur différents arguments dont par exemple les implications économiques du jeune Freud dans l'élaboration de la clinique psychanalytique.

Avec le recul, il me semble assez nettement percevoir que la manière dont Lourau traite de l'institutionnalisation de la psychanalyse résonne fortement avec le regard qu'il porte sur celle de l'analyse institutionnelle. Pour le dire autrement, l'analyse (différée et externe) des implications du jeune Freud par le jeune Lourau invite à l'analyse de celles de ce dernier. L'économie d'ensemble de l'ouvrage L'instituant contre l'institué, qui porte le sous titre "Essais d'analyse institutionnelle" et s'ouvre sur un chapitre en forme de manifeste (ou de programme?) laisse peu de doute sur le fait que cela soit délibéré. S'il s'agit de développer l'analyse institutionnelle, il est nécessaire de se doter d'outils opératoires. Certains sont déjà là, comme les concepts de transfert et de contre-transfert institutionnels produits par le courant de psychothérapie institutionnelle. Ils ont justement pour intérêt principal de mettre en jeu dans les situations analytiques, la diversité des rapports à l'institution.

Le socianalyste qui arrive dans un établissement où il n'était jamais venu, est rapidement assigné à des places, intentions, idéologies, affects, fonctions, statuts... par ses clients. Plutôt que de répondre directement à ce transfert institutionnel (qui parle du rapport des clients à leur institution) il devra mettre en analyse (avec ses coéquipiers voire avec ses clients) ses propres réponses (son contre- 
transfert institutionnel). Ces dernières ayant un rapport direct avec ses propres implications dans sa (ses) propre(s) institution(s).

Ces transfert et contre-transfert institutionnels, ainsi que le "transfert sociologique" que Jacques et Maria van Bockstaele ${ }^{14}$ expérimentent alors, inspirent la pratique de l'intervention socianalytique. Dans un ouvrage rédigé avec Georges Lapassade, Clefs pour la sociologie, la posture du chercheur en situation de travail clinique, le "chercheurpraticien" est décrite dans le cinquième principe de l'intervention socianalytique: ${ }^{15}$

5. L'analyse des implications du chercheur-praticien, c'est-àdire des réponses que le staff-analytique ou le socianalyste font souvent à leur insu à la demande et à la commande. Reconnaître ces implications, c'est faire la plus grande partie du travail socianalytique. Les obstacles à une telle reconnaissance constituent l'essentiel du champ d'analyse. L'implication se formule parfois en termes de "transfert et contre-transfert institutionnels" ; les obstacles à l'analyse, en termes de "résistance". (LAPASSADE ; LOURAU, 1971, p. 200)

Outre le fait qu'il y est question d'un chercheur-praticien, l'analyse de son implication dans la situation d'intervention correspond à celle des réponses qu'il fait aux sollicitations qui lui sont adressées.

En 1981, Lourau donnera une définition plus générale du concept d'implication qu'il applique en dehors du cadre socianalytique. Les implications ne sont pas seulement les "réponses à" mais" l'ensemble des rapports à".

J'appelle implication l'ensemble des rapports que l'intellectuel
refuse, consciemment ou non, d'analyser dans sa pratique,
qu'il s'agisse des rapports à ses objets d'étude, à l'institution
culturelle, à son entourage familial ou autre, à l'argent, au
pouvoir, à la libido et en général à la société dont il fait
partie. Alors que l'intellectuel se croit très capable d'analyser
et d'objectiver ce qui arrive aux autres, y compris, parfois, à
des catégories d'intellectuels dont il s'exclut
d'office. (LAPASSADE ; LOURAU, 1971, p. 200)

Entre les implications qui s'actualisent sur le terrain de l'intervention (et plus généralement à tout travail de recherche sur le terrain) et plus largement les implications du chercheur dans les institutions auxquelles il est lié, une différence va être faite. En 1983, dans la revue POUR $n^{\circ} 88$, Lourau (1988) décline les implications primaires et secondaires:

Implications primaires:

1) implications du chercheur-praticien dans son objet de recherche/intervention; 
2) implication dans l'institution de recherche ou autre institution d'appartenance, et d'abord dans l'équipe de recherche/intervention;

3) implication dans la commande sociale et dans les demandes sociale;

Implications secondaires

4) implications sociales, historiques, des modèles utilisés (implication épistémologique);

5) implications dans l'écriture ou tout autre medium servant à l'exposé de la recherche. (LOURAU, 1988, p. 17)

Continuant à mener simultanément les réflexions théorique et méthodologique, Lourau apporte en 1994 le complément suivant:

Une autre implication secondaire, elle aussi plus extérieure au champ d'intervention - mais non au champ d'analyse défini par les concepts d'implication, de commande, de demandes, d'analyseur, d'autogestion, etc., résiderait dans le choix d'un mode de restitution de l'analyse, par exemple sous la forme d'une monographie à évaluer et analyser collectivement, à diffuser de façon restreinte, à publier [...] (LOURAU, 1994, p. 6-7)

Cette dimension collective, dans l'analyse des implications, est présente dès l'origine dans l'œuvre de Lourau, en particulier par l'idée d'une "autogestion de l'analyse" qui trouve sa pleine expression dans le projet socianalytique qu'il élabore avec Georges Lapassade au milieu des années 1960 à travers une série d'interventions en milieu chrétien.

En $1980^{16}$, mon Lapsus des intellectuels tentait une sociologie de l'intelligentsia centrée sur la notion d'implication. A l'intellectuel organique (de parti ou d'Etat) ou engagé (dans un mouvement idéologique), j'opposais la figure de l'intellectuel impliqué, c'est-à-dire pris dans, ou constitué par son rapport non analysé à l'institution - et cela, qu'il le veuille ou non, qu'il soit organique, engagé ou désengagé. (LOURAU, 1994, p. 10)

Ces formulations: "être pris dans", "être constitué de" renvoient assez directement au terme anglais $d$ ' "involvement" dont on ne trouve pas de traduction suffisamment satisfaisante en français pour la distinguer de "commitment". Dans le chapitre consacré à Dewey, dans le même ouvrage, Lourau se pose d'ailleurs cette question de la traduction en français du terme anglais "involvement " à propos de son étude de Logic: the Theory of Inquiry (1938) ${ }^{17}$.

\section{Analyse interminable}

Dans l'un de ses derniers ouvrages, Lourau revient encore sur l'analyse de l'implication et sur la dimension collective de celle-ci. II montre combien cette analyse est aussi incontournable qu'inépuisable 
(LOURAU, 1997a). Opérant un récapitulatif rapide et très condensé des influences éclectiques dont il est porteur, il propose de penser l'implication à l'aide du concept de transduction. II présente schématiquement une "courbure du concept d'implication" (LOURAU, 1997a, p.35) dans laquelle la "désimplication" et la "surimplication" sont situées dans le domaine de l'inanalysable en situation socianalytique alors que les "appartenances", la "participation" et l'" engagement" sont situés dans celui de l'analysable. Les implications n'étant jamais stabilisées, le déphasage est permanent entre les pôles de la désimplication (hypertrophie de l'objectivité) et de la surimplication (hypertrophie de la subjectivité).

Dans un autre livre paru en 1997b, Lourau explore les incidences du concept de transduction sur l'analyse des implications. La mise en forme de l'ouvrage rompt avec les habitudes dans les publications en sciences sociales. II contient dix variations dans lesquelles interfèrent des approches très diversifiées suivies d'un journal qui occupe la plus grande partie de l'ouvrage. Ce journal, celui de sa recherche interminable sur le concept d'implication, livre les matériaux pour une analyse des implications de l'auteur...

$\mathrm{Si}$, selon les périodes, Lourau a pu insister sur tel ou tel aspect du concept d'implication, la relecture de ses ouvrages fait apparaître une stabilité du concept qui va progressivement s'enrichir par son application à de nouvelles problématiques sans pour autant perdre de sa cohérence. Ce qui fait l'implication, ce sera toujours la complexité des relations que nous entretenons avec les institutions. La mort de Lourau a mis un terme à cette recherche permanente, à cette sorte d'obsession intellectuelle. Mais il faut aussi se souvenir que c'est également à la fin de sa vie, qu'il avait repris une activité socianalytique et qu'il travaillait à une "sociologie du rêve " après avoir mené une étude sur le dispositif de l'assemblée générale (19981999). C'est cette image d'un chercheur en pleine activité intellectuelle et de terrain que je conserve de lui et que je retrouve dans ses écrits.

Par le travail permanent du concept d'implication, Lourau aura lutté contre son institutionnalisation c'est-à-dire contre son enfermement dans une définition définitive. Ce faisant il en aura fait un objet difficile à saisir en une fois, qui pose au chercheur plus de questions qu'il ne lui permet d'en résoudre. L'implication n'a de sens que dans son analyse et celle-ci est nécessairement interminable.

La richesse de cette genèse du concept d'implication chez Lourau, les interférences théoriques et pratiques qui l'ont produit, charge le concept d'une puissance critique dont la mobilisation dans l'activité de recherche est complexe. 


\section{La recherche, un travail institutionnel}

A quoi bon produire des connaissances à l'extérieur de l'institution scientifique si celles-ci sont, de ce fait même, sans effets sociaux? En physique fondamentale comme dans les sciences sociales, les connaissances ne sont pas établies par une découverte mais par un travail institutionnel qui leur permet de s'imposer au sein de la communauté dite scientifique.

La sociologie de la science montre, depuis quelques temps déjà, comment le savoir scientifique est une production qui passe par des opérations matérielles de maniements d'outils techniques (microscopes ou accélérateurs de particules) aussi bien qu'intellectuelles (équations, concepts) et institutionnelles (participation aux organismes scientifiques). Cette sociologie (LATOUR ; WOOLGAR, 1989) ${ }^{18}$ montre aussi que c'est par un travail institutionnel intensif au sein des institutions d'Etat, comme des associations de chercheurs, qu'un savoir toujours discuté et discutable est produit. Le fait qu'il soit discutable est d'ailleurs, très classiquement, la condition de la scientificité.

\section{Références Bibliographiques}

CABET, É. Voyage en I carie. Paris: J. Mallet et cie, 1842.

CHEVILLION, B. Lucien Bonnafé, psychiatre désaliéniste. Paris: L'Harmattan, 2005.

CROZIER, M.; FRIEDBERG, E. L'acteur et le système: les contraintes de l'action organisée. Paris: Seuil, 1977.

DEWEY, J. Logique : la théorie de l'enquête. Paris: PUF, 1967. FOUCAULT, M. Le corps utopique. Les hétérotopies. Paris: Ligne, 2009.

FREUD, S. (1921). Psychologie des masses et analyse du moi. In: $\overline{1} \overline{\overline{5}} \overline{\text {. }}$ . Essais de Psychanalyse. Paris: Payot, 1981, v. XVI, p. 80-

GABEL, J. La fausse conscience. Paris: Minuit, 1962.

LAMIHI A.; MONCEAU, G. Institution et implication: l'œuvre de René Lourau. Paris: Syllepse, 2002.

LAPASSADE G.; LOURAU, R. Clefs pour la Sociologie. Paris: Seghers, 1971.

LATOUR, B.; WOOLGAR, S. La vie de laboratoire: la production des faits scientifiques. Paris: La Découverte, 1989.

LE BON, G. (1985) Psychologie des foules. Paris: Alcan, 1963.

LEFEBVRE, H. Le langage et la société. Paris: Gallimard, 1966.

LOURAU, R. Le poète devant les institutions. La tour de feu, Paris, n. 82, p.123-129, 1964.

L'instituant contre l'institué: essais d'analyse

institutionnelle. Paris, Anthropos, 1969. 
.L'autogestion instituée. In : LAPASSADE, G. (Dir.). L'autogestion pédagogique. Paris: Gauthiers-Villars, 1971a, p. 99111.

Un problème politique? In: LAPASSADE, G. (Dir.). L'autogestion pédagogique. Paris: Gauthiers-Villars, 1971b, p. 1934.

. A análise institucional. Petrópolis: Vozes, 1975.

L'etat-inconscient. Paris: Minuit, 1978.

Autodissolution des avant-garde. Paris: Galilée, 1980.

Le lapsus des intellectuels. Toulouse: Privat, 1981.

- Quelques approches de l'implication suivi de "Genèse du concept d'implication". POUR, Paris, n. 88, p.12-27, 1988.

Actes manqués de la recherche. Paris: P.U.F., 1994.

La clé des champs: une introduction à l'analyse institutionnelle. Paris: Anthropos, 1997a.

I mplication et transduction. Paris: Anthropos, 1997b.

MAC DOUGALL, J. A note on suggestion, J ournal of Neurology and Psychopathologie, London, v. 1, n. 1, p. 1-10, mai. 1920.

MICHAUD, G. Laborde un pari nécessaire. Paris: Bordas, 1977.

MONCEAU, G. Actes manqués de la recherche. In: LAMIHI A.; MONCEAU, G. Institution et implication: l'œuvre de René Lourau. Paris: Syllepse, 2002, p. 161-170.

Como as instituições permeiam as práticas profissionais: sócioclínica institucional e formação de professores. In : PIMENTA, S. G.; SANTORO, M. A. F. (Orgs.). Pesquisa em educação: possibilidades investigativas/ formativas da pesquisa-ação. São Paulo: Ed. Loyola, 2008a, v. 1, p. 27-73.

. Implicação, sobreimplicação e implicação profissional. Fractal

- Revista de Psicologia, v. 20, n. 1, p. 19-26, jan./jul. 2008 b.

REVON, Alain. Note bibliographique sur L'institué contre l'instituant. Revue Française de Sociologie, v. 11, 1970, p. 432-433.

SAVOYE, A. Pour une analyse institutionnelle du parti. L'Homme et la Société, Paris, n. 29-30, p. 107-114, 1973.

VAN BOCKSTAELE, J.; VAN BOCKSTAELE, M. La socianalyse. Paris: Anthropos, 2004.

Endereço para correspondência

Gilles Monceau

2 rue de la Liberte. 93526, Saint-Denis Cedex, Paris, França

Endereço eletrônico: Gilles.Monceau@univ-paris8.fr

Recebido em: 02/08/2009

Aceito para publicação em: 10/08/2009

Acompanhamento do processo editorial: Deise Mancebo, Marisa Lopes da Rocha e Roberta Romagnoli 


\section{Notas}

${ }^{1}$ Les sauvages étant toujours ceux qui ne partagent pas les mêmes institutions que nous et que nous parvenons à dominer en leur imposant les nôtres (Eglise, Etat, entreprises multinationales, ONG...).

${ }^{2}$ Voir par exemple l'édition récente d'un recueil de textes de Lucien Bonnafé, dont des lettres, qui témoignent des débuts de ce travail institutionnel : Bernadette Chevillion (textes rassemblées par), Lucien Bonnafé, psychiatre désaliéniste, Paris, L'Harmattan, 2005.

${ }^{3}$ René Lourau, "L'autogestion instituée ", in G. Lapassade (dir.), L'autogestion pédagogique, Paris, Gauthiers-Villars, 1971a, pp. 99-111. Le texte écrit en 1964 est publié en 1970 dans cet ouvrage collectif.

4 Sigmund Freud, "Psychologie des masses et analyse du moi (1921) ", Essais de psychanalyse, Paris, Payot, trad. fr. S. Jankélévitch, 1981, p.150. La traduction dont disposait Lourau avait bien alors pour titre "Psychologie collective et analyse du moi ".

${ }^{5}$ Gustave Le Bon, Psychologie des foules, Paris, Alcan, 1895 (réed. 1963)

${ }^{6}$ Michel Crozier et Ehrard Friedberg publieront quelques années plus tard : L'acteur et le système. Les contraintes de l'action organisée, Paris, Seuil, 1977.

7 René Lourau, A Análise Institucional, Op. cit. pp. 272-273 (pagination de la version française initiale parue en 1970 aux éditions de Minuit)..

${ }^{8}$ Intérêt pour une sociologie des mouvements littéraires déjà signalé avec "Le poète devant les institutions".

${ }^{9}$ Relisant ce texte en 2009, je m'aperçois que j'avais conservé un autre souvenir de l'image proposée par Lourau. Dans ma mémoire, c'était l'institution qui parlait par le ventre en parlant par la bouche de l'individu qui se trouvait au-dedans d'elle! Ce renversement de l'image n'altère pas l'idée selon laquelle l'institution habite les individus mais m'interroge tout de même...

10 Et Lourau fait ici référence au Phénomène bureaucratique (1963) de Michel Crozier.

${ }^{11}$ Compte chèque postal

${ }^{12}$ Revue française de sociologie, vol. 11, 1970

${ }^{13}$ Dans lequel se trouve "Psychologie collective et analyse du moi " précédemment cité.

${ }^{14}$ Ceux-ci sont récemment revenus sur leur propre cheminement dans : Jacques et Maria Van Bockstaele, La socianalyse, Paris, Anthropos, 2004.

${ }^{15}$ II a été fait référence à ces principes dans la seconde partie de cette note.

${ }^{16}$ Le livre n'a été publié qu'en 1981.

17 Traduction française: Logique: la théorie de l'enquête, Paris, PUF, 1967 (réed., 1993).

${ }^{18}$ Bruno Latour et Steve Woolgar, La Vie de laboratoire. La production des faits scientifiques, Paris La Découverte, 1989 (1ère éd. anglaise : 1979). 